\title{
Controversias etiológicas, diagnósticas y terapéuticas de la periodontitis agresiva
}

\author{
DISCEPOLI N* \\ BASCONES MARTÍNEZ A**
}

Discepoli N, Bascones-Martínez A. Controversias etiológicas, diagnósticas y terapéuticas de la periodontitis agresiva. Av Periodon Implantol. 2008; 20, 1:39-47.

\section{RESUMEN}

Se revisa la bibliografía más reciente al resguardo de la Periodontitis Agresiva, con particular atención a la etiología multifactorial, al diagnóstico, clínico y de laboratorio, y a la fase terapéutica. Se revisan también las controversias entre las diferentes clasificaciones que se propusieron hasta hoy.

\section{PALABRAS CLAVE}

Periodontitis agresiva. Patogenia. Clasificación. Diagnostico diferencial. Modalidades de tratamiento.

Fecha de recepción: Noviembre 2007.

Fecha de aceptación: Diciembre 2007.

\section{INTRODUCCIÓN}

Las periodontitis agresivas engloban un grupo de periodontitis raras, frecuentemente severas y de progresión rápida caracterizadas la mayoría de las veces por manifestarse clínicamente en edades tempranas.

Hasta hoy, catalogar dentro de las periodontitis agresivas una periodontitis, continua siendo un aspecto controvertido a la hora de diagnosticar las diferentes enfermedades periodontales. Esto se debe fundamentalmente a que éste tipo de periodontitis ha estado definido basado en diferentes factores que han ido modificándose a lo largo del tiempo en función de las diferentes clasificaciones que han ido apareciendo.

En este trabajo, vamos a intentar definir y entender lo que hoy día es considerado como "periodontitis agresiva" partiendo de los estudios más relevantes que empezaron a definirlas, repasando algunos conceptos de su epidemiología, profundizando en las características que hoy día se consideran que la definen y comentando algunos aspectos sobre su diagnóstico y terapéutica.

\section{CLASIFICACIÓN PREVIA}

En 1989, el World workshop en periodoncia clínica, propuso una nueva clasificación de las enfermedades periodontales, basada en el paradigma infección-huésped. Sin embargo, esta misma clasificación depende todavía de manera muy evidente de la edad de los pacientes y de la tasa de progresión.

I. Periodontitis del adulto.

II. Periodontitis de comienzo temprano.

A. Periodontitis prepuberal.
1. Generalizada.
2. Localizada. 
B. Periodontitis juvenil.

1. Generalizada.

2. Localizada.

C. Periodontitis rápidamente progresiva.

III. Periodontitis asociada con enfermedades sistémicas.

IV. Periodontitis ulcerativa necrotizante.

V. Periodontitis refractaria.

\section{CLASIFICACIÓN Y SUS PROBLEMAS}

El término "periodontitis agresiva" (P.A.) fue propuesto por primera vez en 1999 para sustituir al término anterior de periodontitis de comienzo temprano. Este último fue utilizado en las clasificaciones de 1989 (AAP) y la de 1993 (European classification) como un conjunto de enfermedades periodontales destructivas que afectan a pacientes jóvenes.

El mayor problema en la clasificación de 1989 fue su arbitraria y fuerte dependencia con la edad de los pacientes afectados o con la edad de comienzo de la enfermedad. La periodontitis del adulto y la periodontitis de comienzo temprano estaban basadas firmemente en la edad como un criterio para clasificar a los pacientes dentro de una categoría o de otra. En esta clasificación, la línea divisoria entre adulto y comienzo temprano era arbitraria, a los 35 años. De todas formas, las características clínicas del paciente eran importantes (por ejemplo, incisivos/primeros molares afectados en la periodontitis juvenil localizada), pero las decisiones para el diagnóstico final dependían mucho de la edad del paciente.

Cuando se usa la edad como el único y más importante determinante en la clasificación, aparecen preguntas y dudas. ¿Es necesario establecer la edad de comienzo de la periodontitis antes de que un paciente pueda ser correctamente clasificado o diagnosticado?, conforme un niño o adolescente con periodontitis crece ¿debería cambiarse el diagnóstico periodontal (es decir, con el tiempo la periodontitis prepuberal llega a ser periodontitis rápidamente progresiva)? Sin embargo, se puede pensar que es incorrecto o simplemente erróneo usar la edad como el criterio principal para asignar los diferentes nombres de la periodontitis que afectan a varios miembros de una familia. De hecho, es más probable que las tres subcategorías de la periodontitis de comienzo temprano sean la misma enfermedad en vez de tres formas separadas de periodontitis.

De todas las razones para cuestionarse el uso de la edad como criterio de clasificación, la más convincente son los datos de los estudios epidemiológicos que indican que niños y adolescentes desarrollan pérdida de inserción con un tipo de periodontitis que clínicamente se parece a la vista en los adultos. En otras palabras, ciertos niños y adolescentes desarrollan una enfermedad idéntica a la periodontitis del adulto excepto que los afectados no son adultos.

De hecho se sugirió que el diagnóstico de cualquiera de las formas de periodontitis no debería basarse en la edad del paciente, sino más bien en los hallazgos clínicos, radiológicos, de anamnesis, y de laboratorio (Consensus paper AAP 1999).

En términos generales, la nueva designación de periodontitis agresiva localizada (P.A.L.) reemplazaría al viejo término de "periodontitis juvenil localizada", y la nueva designación de periodontitis agresiva generalizada (P.A.G.) reemplazaría al de "periodontitis juvenil generalizada". Se concluyó que la P.A. es un tipo específico de periodontitis con características clínicas y hallazgos de laboratorio claramente identificables, lo cual hace que sea suficientemente diferente de la periodontitis crónica (P.C.) como para garantizar una clasificación separada.

En el Workshop del 1999 de la AAP, se definen algunas características que ayudan al diagnóstico y al diagnóstico diferencial entre la forma generalizada y la localizada; pero que quede claro que no se puede transferir de manera directa las informaciones de las viejas clasificaciones (1989-1993) hacia la nueva. Por ejemplo, pacientes previamente clasificados como periodontitis juvenil generalizada, podrían ser clasificados como P.C. o como P.A.G., según la nueva clasificación, y según las características primarias o secundarias del sujeto (1).

La periodontitis prepuberal (PP), que aparecía en la clasificación del 1989, fue la primera eliminada. Muchos de los pacientes en esta categoría resultaban que tenían o hipofosfatasia o deficiencia en la adhesión del leucocito (síndrome LAD). Es decir, es probable que la mayoría de los niños prepuberales con destrucción periodontal severa que afecte a la dentición primaria probablemente tengan una enfermedad sistémica que aumente su susceptibilidad a las infecciones bacterianas como: síndrome LAD, inmunodeficiencia primaria congénita, defectos crónicos del neutrófilo y neutropenia cíclica. Estos pacientes tendrían que ser apropiadamente colocados bajo la categoría general de "periodontitis como manifestación de enfermedades sistémicas". Los pacientes de esta edad, sanos, se reparten, según las características, en P.C. o en P.A. 
La periodontitis rápidamente progresiva (PRP) se ha sido eliminado también de la clasificación (1999), porqué aparecía como una serie de enfermedades demasiado heterogéneas. Por ejemplo, los clínicos tienen que documentar que ha ocurrido una rápida progresión antes de dar a un paciente este diagnóstico, para ser designado como rápido, ¿cuánta progresión tiene que ocurrir y en cuanto tiempo?. ¿Se puede asumir de un solo examen clínico que un adolescente $o$ un joven adulto con gran pérdida de inserción tiene esta enfermedad?.¿Cómo puede el clínico distinguir entre una "periodontitis juvenil generalizada" y una "periodontitis rápidamente progresiva"?.Ya que no hay respuestas definitivas a estas y a otras cuestiones similares, la clasificación perdió alguna utilidad clínica. Casi cualquier forma de periodontitis puede progresar rápida o lentamente dependiendo del conjunto de circunstancias que gobiernen la naturaleza de las interacciones huésped-bacteria durante un período de tiempo. Así, los pacientes que habían sido clasificados en este grupo se asignan o al grupo de P.A.G. o al grupo de P.C., según sus características.

\section{DEFINICIÓN Y CARACTERÍSTICAS}

Según la clasificación del "Internacional Workshop" de 1999, la periodontitis agresiva (P.A.) se clasifica sencillamente en dos formas distintas:

- Periodontitis agresiva localizada (P.A.L.)

- Periodontitis agresiva generalizada (P.A.G.)

La nueva designación de P.A.L. reemplazaría al viejo término de "Periodontitis Juvenil Localizada" (AAP 1989), y la definición de P.A.G., al de "Periodontitis Juvenil Generalizada". (AAP 1989).

Las características primarias o comunes para la P.A. son (figuras 1 a 4):

- Los pacientes, excepto por la presencia de periodontitis, están clínicamente sanos.

- Rápida pérdida de inserción y rápida destrucción de hueso.

- Agregación familiar.

Las características secundarias que son generales, pero no universales, son:

- La cantidad de placa bacteriana no es coherente con la cantidad de destrucción periodontal.
- Proporciones elevadas de Aggregatibacter actinomycetemcomitans, y en algunas poblaciones Porphyromonas gingivalis puede estar elevada.

- Deficiencias fagocíticas.

- Hiper-respuesta de macrófagos, capaces de inducir elevados niveles de IL- $1 B$ y de PGE $_{2}$.

- La progresión de la pérdida de inserción y de hueso puede ser autolimitante.

Un punto fundamental es que no todas estas características secundarias tienen que estar presentes a la vez, para que sea asignada la definición de P.A.

Además de estas características en común para las dos formas de P.A, hay algunas estrictamente específicas para cada una:

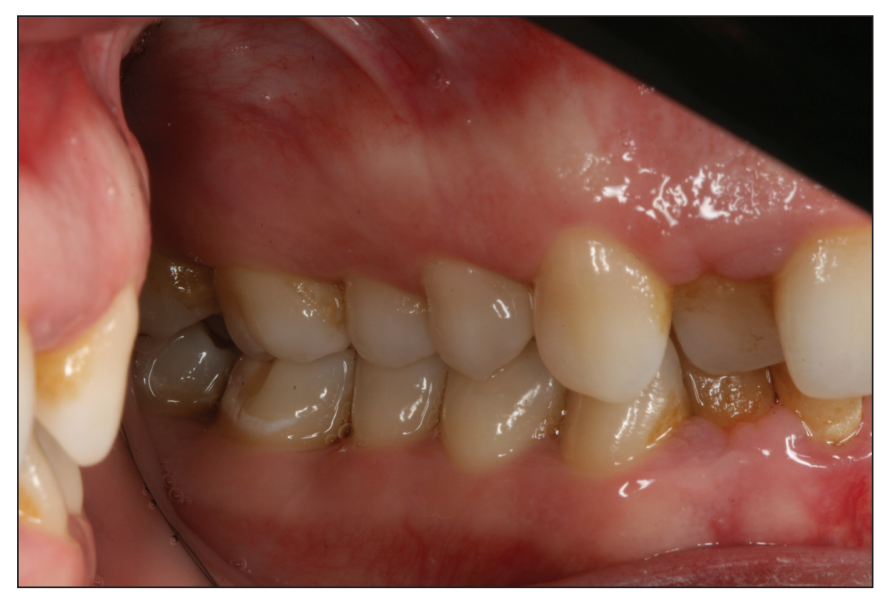

Fig.1.

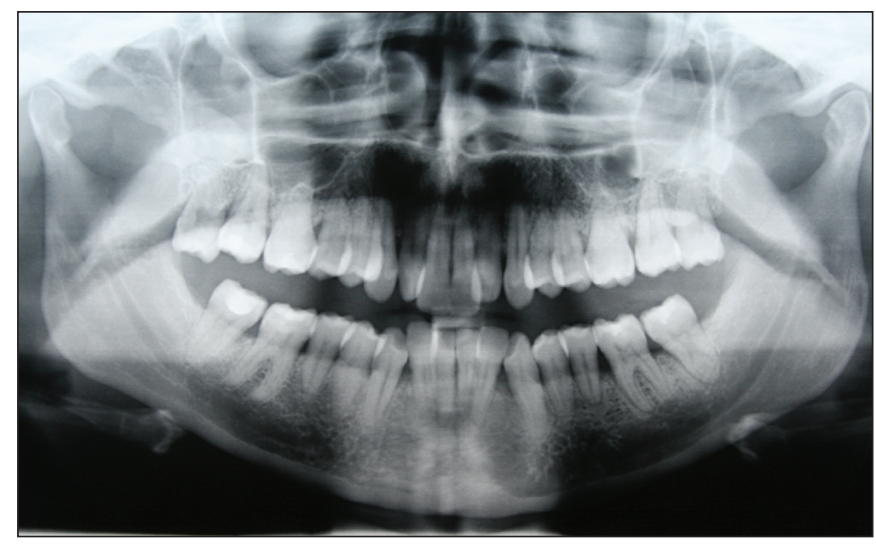

Fig.2. 


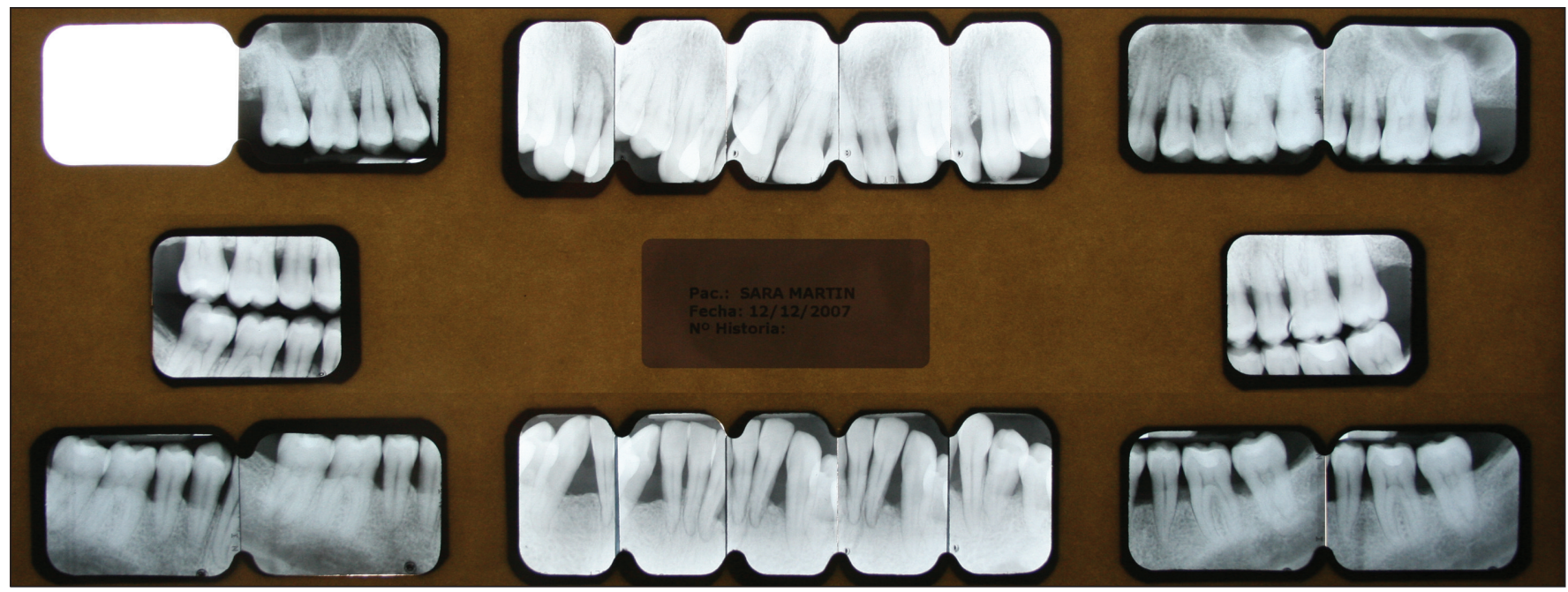

Fig.3.

\section{PERIODONTITIS AGRESIVA LOCALIZADA}

1. Comienzo circumpuberal.

2. Localizada en primeros molares/incisivos, con pérdida de inserción interproximal en al menos dos dientes permanentes, uno de los cuales es un primer molar, y que afecta a no más de otros dos dientes, aparte de los primeros molares e incisivos.

Pero puede presentar también patrones atípicos.

3. Se asocia frecuentemente con $A$. actinomycetemcomitans.

4. Se asocia frecuentemente con deficiencias en la función neutrófila.

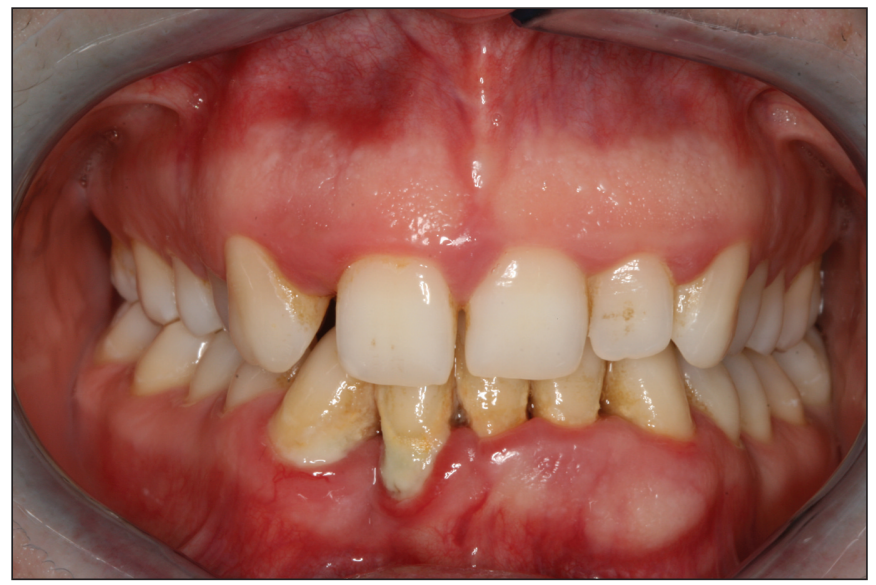

Fig.4.
5. Se asocia frecuentemente con una respuesta elevada de anticuerpos séricos contra los patógenos.

\section{Periodontitis agresiva GeNERALIZADA}

1. Normalmente afecta a sujetos por debajo de los 30 años, pero pueden ser mayores

2. Pérdida de inserción interproximal generalizada, que afecta como mínimo a tres dientes permanentes además de los primeros molares e incisivos.

3. Se asocia frecuentemente con $A$. actinomycetemcomitans y $P$. gingivalis.

4. Se asocia frecuentemente con deficiencias en la función neutrófila.

5. Se asocia frecuentemente con una baja respuesta de anticuerpos séricos contra los patógenos

6. Forma de pérdida de inserción y de hueso de naturaleza episódica.

NotA: A esta clasificación se le pueden añadir descriptores secundarios como factores modificadores del riesgo (tabaco, estrés, drogas, hormonas, etc.) que pueden modificar y afectar el curso de todos los tipos de periodontitis.

(Consensus Report, Ann Periodontol, 1999)

\section{EPIDEMIOLOGÍA}

No hay muchos estudios al respecto de la prevalencia de la P.A., pero dentro de los que hay se puede notar 
claramente que este tipo de enfermedad puede manifestarse en cualquier tipo de edad y de raza (2). Sin embargo hay mucha variabilidad de resultados, debida probablemente a diferencias en la metodología epidemiológica utilizada, y a la definición.

\section{Dentición Primaria:}

El porcentaje de prevalencia en jóvenes de 5-11 años varía entre el 0,9 y el $4,5 \%(3,4)$.

\section{DENTICIÓN SECUNDARIA:}

La mayoría de los estudios, con sujetos entre 13 y 20 años, observan una prevalencia siempre menor del $1 \%$ (en población caucasiana entre 0,1 y $0,2 \%$ ) (2). El riesgo de padecer la enfermedad no parece ser compartido igualmente entre distintas razas: en un estudio sobre la población escolar de EEUU, de 5-12 años, la prevalencia fue de $0,2 \%$ en Caucasianos y de $2,6 \%$ en Afroamericanos (5). En un estudio que analizó un cohorte de pacientes Afroamericanos entre 18 y 35 años de edad, siempre en EE.UU., el porcentaje alcanzó el $3,6 \%(6)$.

\section{FACTORES DE RIESGO PARA LA PERIODONTITIS AGRESIVA}

La Academia Americana de periodoncia resume esquemáticamente la patogénesis de las periodontitis y la influencia que determinados factores de riesgo ejercen sobre ella como lo observamos en la figura $5(7)$ :

\section{BACTERIAS ESPECÍFICAS}

Muchas bacterias se han hallado frecuentemente en sujetos con P.A.L. (A. actinomycetemcomitans; especies de Capnocytophaga; Eikenella corrodens; Prevotella intermedia, Campylobacter rectus). Sin embargo, A. actinomycetemcomitans se ha implicado como el principal patógeno asociado con esta enfermedad.

Según Tonetti y Mombelli, (8), esta asociación esta basada en estas consideraciones:

- A. actinomycetemcomitans se encuentra en un alto número de lesiones de P.A.L. (90\%).

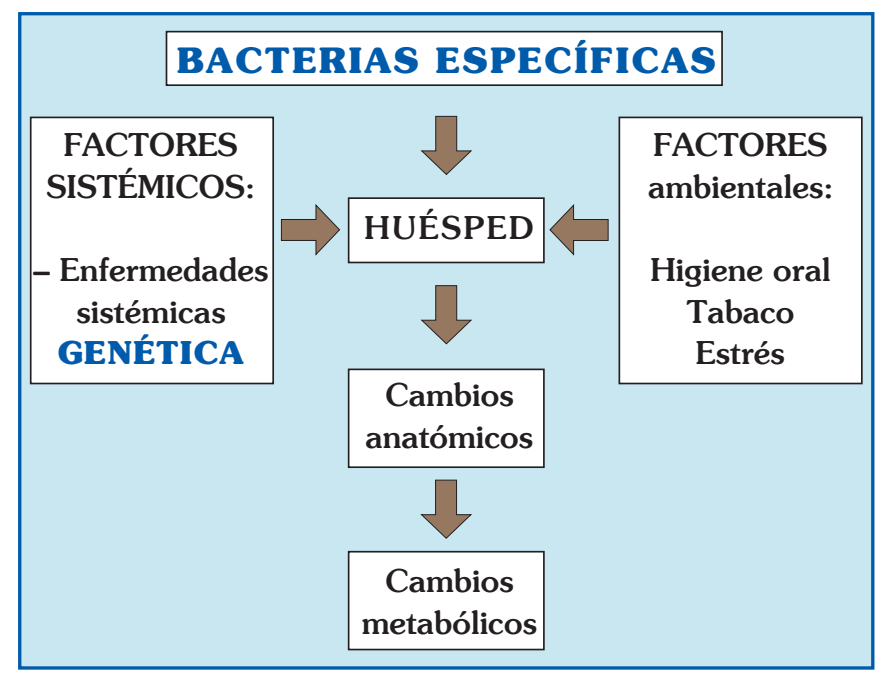

Fig.5.

- Localizaciones con evidencias de progresión activa de la enfermedad presentan $A$. actinomycetemcomitans.

- Elevados títulos de anticuerpos específicos contra A. actinomycetemcomitans, en pacientes con signos clínicos de P.A.L.

- En estudios clínicos, la reducción de la carga subgingival de $A$. actinomycetemcomitans durante la fase de tratamiento se ha asociado con un éxito del tratamiento.

- A. actinomycetemcomitans produce una cantidad de factores de virulencia que pueden contribuir al desarrollo de la enfermedad.

El estudio de Haubek 2002 (9) demuestra que la presencia de la cepa altamente leucotóxica de A. actinomycetemcomitans (JP2) supone un incremento en el riesgo de pérdida de inserción. Los autores realizaron el estudio en adolescentes con P.A.L. y observaron que los pacientes positivos para la cepa JP2 presentaban un mayor número de dientes con pérdida de inserción y mayor cantidad de pérdida de inserción que los pacientes que no tenía esta cepa. A los dos años, éste mismo equipo (10), observó que la presencia de este clon era predictiva para la pérdida progresiva del nivel de inserción, con una tasa de probabilidad (OR) de 14,5.

No todas las investigaciones encuentran una asociación entre $A$. actinomycetemcomitans y la P.A. Actualmente, la mera presencia de $A$. actinomycetemcomitans no es tenida en cuenta a la hora de diferenciar entre forma crónica y agresiva de periodontitis (11). Esta revisión sistemática nos indica claramente que la pre- 
sencia de bacterias, aunque especificas, no nos permite efectuar un diagnostico diferencial correcto. Otros estudios, como el de Wang y cols. en 2005 (12), han demostrado, tras el análisis de muestras de placa subgingival, que la presencia de $A$. actinomycetemcomitans en sujetos con PC y PAG generalizada era similar (25\% y $24 \%$ respectivamente).

\section{GENÉTICA Y ALTERACIONES DEL HUÉSPED}

Las bacterias periodontopatógenas constituyen el agente etiológico primario de las periodontitis. De todas formas, el riesgo de padecer la enfermedad periodontal no es el mismo para todos los pacientes, hecho que sugiere que también las características del huésped están involucradas en definir un patrón de susceptibilidad a la enfermedad.

\section{SISTEMA DE HERENCIA DE LA PERIODONTITIS AGRESIVA (AGREGACIÓN FAMILIAR)}

Mucha de la evidencia disponible que avala la predisposición genética a esta enfermedad, se debe al análisis de segregación en familias con sujetos que padecen P.A y que sigue manifestándose desde dos o más generaciones. Los resultados son muy distintos, con patrones de transmisión autosómica dominante, autosómica recesiva y dominante ligado a X; todavía no ha sido posible establecer un único patrón que incluyera a todas las familias.

\section{Polimorfismos}

Se han investigado muchos polimorfismos con el objetivo de descubrir un posible marcador de susceptibilidad para la P.A., entre otros:

- Interleukina-la

- Interleukina-4

- Interleukina-10

- TNF- $\alpha$

- Receptores-FC

- HLA

- Receptor-vitD

- Receptor N-formylpeptide.

Los estudios sobre la relación entre estos genes y la susceptibilidad a la P.A. en diferentes poblaciones han observado resultados contradictorios. Algunos estu- dios han evidenciado el efecto combinado de algún polimorfismo y el sexo o el tabaco en términos de susceptibilidad. Estos resultados nos sugieren la existencia de una heterogeneidad genética en la P.A.

\section{DEFECTOS DE LOS NEUTRÓFILOS EN LA PERIODONTITIS AGRESIVA}

Los polimorfonucleares neutrófilos son el principal componente de la defensa innata humana. Las funciones defectuosas de esta línea celular están involucradas en una mayor susceptibilidad a la periodontitis, con frecuentes episodios de destrucción periodontal. Las anormalidades mas estudiadas son:

- Adhesión.

- Quimiotaxis.

- Producción de superóxido.

- Fagocitosis.

- Actividad bactericida.

\section{Adhesión}

El antígeno-l asociado a la función de los leucocitos y el receptor para el factor del complemento $n^{\circ} 3$ participan en la adhesión de los granulocitos. Los dos receptores parecen estar anormales en pacientes con periodontitis prepuberal generalizada, mientras se encuentran normales en pacientes sanos, con periodontitis crónica y con la forma localizada de la periodontitis prepuberal ((13). De hecho, muchos de los pacientes diagnosticados como periodontitis prepuberal generalizada presentaban una deficiencia en la adhesión de los leucocitos (síndrome LAD).

\section{Quimiotaxis}

Hay una correlación entre la disminución del número de receptores para el péptido $\mathrm{N}$-formil-metionil-leucilphenilalanina y la interleuquina-8 (14) y el desarrollo de formas de periodontitis juvenil localizada. La misma relación se puede encontrar con la depleción del factor $n^{\circ} 5$ a del complemento y la periodontitis juvenil localizada (15).

\section{Defectos quimiotácticos}

Kinane y cols., (16) no consiguieron encontrar ninguna alteración de la movilidad en los neutrófilos de pa- 
cientes de raza caucasiana del norte de Europa. Takahashi y cols., (17) confirman la ausencia de cualquier relación entre pacientes con periodontitis de comienzo temprano (de forma generalizada y localizada) y las alteraciones de los neutrófilos.

Sin embargo, en los estudios realizados en Estados Unidos, aproximadamente un $60-70 \%$ de los pacientes con P.A.L. mostraron una reducción en la movilidad de los neutrófilos. $(18,19)$. También se ha caracterizado esta alteración en las familias de raza afroamericana esta (15). Estas diferencias étnicas, podrían soportar la hipótesis de que haya distintas manifestaciones de periodontitis agresiva, pero con la misma presentación clínica.

\section{INMUNOLOGÍA}

En un estudio reciente llevado a cabo por Picolos y cols., (20) se ha observado que los títulos de anticuerpos no estaban relacionados con el diagnóstico de la forma generalizada o localizada de la P.A. De hecho, los pacientes con P.A.L. presentaban títulos de anticuerpos ligeramente más bajos que los pacientes con la forma generalizada para la mayoría de las especies bacterianas estudiadas, incluyendo $A$. actinomycetemcomitans, $P$. gingivalis y Tannerella forsythia.

Hoy en día se sabe que muchos factores de riesgo para la periodontitis pueden influir sobre la producción de anticuerpos. Entre otros, la edad, el sexo, la raza, el tabaco y el estrés, $(21,22)$.

\section{DIAGNÓSTICO}

El objetivo del diagnóstico clínico es detectar pacientes con P.A. y de identificar los factores que modifican el tratamiento.

La primera pregunta que el clínico tiene que hacer es:

\section{1) ¿Hay periodontitis?}

Una respuesta adecuada conlleva la recopilación sistemática de la historia clínica para responder a los siguientes aspectos:

- ¿Hay pérdida de soporte periodontal? (Pérdida de inserción y reabsorción de hueso).
- ¿Dicha pérdida de inserción está asociada a recesiones o a bolsas?

- ¿Hay otra causa para la pérdida de inserción que no sea la periodontitis?

- ¿Hay otro proceso que imita a la periodontitis a través de la formación de pseudobolsas?

Antes de decir si la pérdida de inserción que tenemos es periodontitis, hay que tener en cuenta que en muchos sujetos (sobretodo jóvenes), se puede observar pérdida de inserción debida a causas traumáticas, a la presencia de dientes impactados, a causa de movimientos ortodóncicos o de márgenes subgingivales. Todas estas situaciones pueden producir pérdida de inserción, que pero se debe de diagnosticar no como periodontitis, sino más bien como:

\section{"pérdida de inserción incidental"}

Una vez que tenemos claro que estamos frente a una periodontitis, el segundo paso es preguntarnos:

\section{2) ¿Hay una condición sistémica que pueda por si sola justificar esta periodontitis?}

Si la respuesta es sí, estamos frente a una periodontitis como

\section{"manifestación de enfermedades sistémicas"}

Si la respuesta es no, el siguiente paso es:

\section{3) ¿Hay signos o síntomas de una periodontitis ne- crotizante?}

Si la respuesta es no, hemos llegado al momento decisivo en que debemos hacer el diagnóstico diferencial entre P.C. y P.A. Según Armitage (1999), el diagnóstico de P.A. se hace excluyendo la presencia de la forma crónica, que es la forma mas frecuente de enfermedad periodontal. Sin duda alguna, la ayuda más importante es hacer referencia a las tres características principales comunes que describen la enfermedad (Tonetti 99). Estas características sin embargo, incluyen parámetros clínicos y de laboratorio; estos últimos sirven frecuentemente solo como confirmación de un diagnostico clínico previamente hecho.

¿Sobre qué elementos nos basamos para hacer un diagnóstico clínico de P.A? 
- Una rápida destrucción del periodonto es el mayor criterio diagnóstico de P.A, y aunque la correcta aplicación de este criterio requiera la presencia de datos clínicos y radiográficos tomados en distintos momentos, la presencia de una destrucción severa en relación con la edad, es suficiente para definir una rápida progresión.

Nota aclaratoria: posteriormente a 1999, varios autores han cuestionado este punto y han defendido la necesidad de dos registros clínicos y radiográficos tomados en distintos momentos y que evidencien la rápida progresión de la enfermedad para hacer el diagnóstico de P.A.

- En la mayoría de los casos de P.A, la cantidad de destrucción periodontal parece ser mayor de la esperada por el simple acumulo de factores locales. Este criterio, aunque no siempre verdadero, nos indica o la presencia de una flora patógena particularmente virulenta o la presencia de un huésped muy susceptible.

El artículo de consenso (AAP 1999) concluye que no todas las características primarias y secundarias tienen que estar presentes para asignar un diagnóstico de periodontitis agresiva y que el diagnóstico puede basarse exclusivamente en datos clínicos, radiográficos e históricos.

Nota aclaratoria: Hoy en día se piensa que es imprescindible que se den las 3 características primarias para hacer el diagnóstico de P.A., si alguna de ellas falla se hará el diagnóstico de P.C.

\section{DIAGNÓSTICO RADIOGRÁFICO}

La importancia de esta ayuda se refleja sobretodo en sujetos jóvenes, con dentición mixta. En estos pacientes el sondaje puede resultar confuso, entonces el uso de aletas de mordida puede resultar esencial. Una distancia mayor de $2 \mathbf{~ m m}$ entre la línea amelocementaria y la cresta alveolar en sujetos con dentición mixta nos debe hacer sospechar de la presencia de P.A (3).

\section{TRATAMIENTO}

La AAP desarrolló una serie de parámetros a seguir a la hora de tratar las P.A. (23). Puntualizaron lo siguiente:

- El objetivo del tratamiento es el mismo que para las P.C.: disminuir o eliminar la carga bacteriana y los factores de riesgo contribuyentes, y regenerar el aparato de inserción siempre y cuando esté indicado.
— Los métodos de tratamiento son similares a los de la periodontitis crónica, pero además debe considerarse lo siguiente:

1. Evaluación sistémica del paciente para descartar enfermedades, sobretodo en niños y adolescentes.

2. El tratamiento mecánico básico como único tratamiento suele ser inefectivo.

3. Los resultados del tratamiento a largo plazo dependerán de la implicación del paciente y de los intervalos de los mantenimientos. Cuando estén afectados los dientes temporales, los dientes permanentes deben ser monitorizados para detectar posibles futuras pérdidas de inserción.

4. Debido a la asociación familiar, se recomienda el asesoramiento y evaluación de todos los miembros de la familia del afectado.

- Los resultados esperados tras el tratamiento periodontal de las P.A. debería incluir:

- Reducción importante de los signos de la inflamación gingival

- Reducción de la profundidad de sondaje

- Ganancia o estabilidad del nivel de inserción

- Evidencia radiográfica de la resolución de las lesiones óseas.

- Reducción de la placa clínicamente detectable compatible con un nivel de salud periodontal.

- Progreso hacia una estabilidad oclusal.

\section{SUMIMARY}

In this paper is reviewed the most recent bibliography about Aggressive Periodontitis, with particular attention on its multifactor aetiology, on diagnostic, clinic and laboratory, and on the therapeutic phase. The paper will review too the controversies among classifications proposed up to the date.

\section{KEY WORDS}

Aggressive Periodontitis. Etiopathogenia. Classification. Diferencial Diagnosis. Treatment modalities.

\section{BIBLIOGRAFÍA}

1. Armitage GC. Development of a classification system for periodontal diseases and conditions. Annals of periodontology/the American Academy of Periodontology. 1999 Dec;4(1):1-6. 
2. Papapanou PN. Periodontal diseases: epidemiology. Annals of periodontology / the American Academy of Periodontology. 1996 Nov;1(1):1-36.

3. Sjodin B, Matsson L, Unell L, Egelberg J. Marginal bone loss in the primary dentition of patients with juvenile periodontitis. Journal of clinical periodontology. 1993 Jan;20(1):32-6.

4. Sweeney EA, Alcoforado GA, Nyman S, Slots J. Prevalence and microbiology of localized prepubertal periodontitis. Oral microbiology and immunology. 1987 Jun;2(2):65-70.

5. Loe H, Brown LJ. Early onset periodontitis in the United States of America. Journal of periodontology. 1991 Oct;62(10):608-16.

6. Oliver RC, Brown LJ, Loe H. Periodontal diseases in the United States population. Journal of periodontology. 1998 Feb;69(2):269-78.

7. AAP. Position paper (AAPa):The pathogenesis of periodontal dideases. j periodontol. 1999;70:457-70.

8. Tonetti MS, Mombelli A. Early-onset periodontitis. Annals of periodontology / the American Academy of Periodontology. 1999 Dec;4(1):39-53.

9. Haubek D, Ennibi OK, Abdellaoui L, Benzarti N, Poulsen S. Attachment loss in Moroccan early onset periodontitis patients and infection with the JP2-type of Actinobacillus actinomycetemcomitans. Journal of clinical periodontology. 2002 Jul;29(7):657-60.

10. Haubek D, Ennibi OK, Poulsen K, Benzarti N, Baelum V. The highly leukotoxic JP2 clone of Actinobacillus actinomycetemcomitans and progression of periodontal attachment loss. J Dent Res. 2004 Oct;83(10): 767-70.

11. Mombelli A, Casagni F, Madianos PN. Can presence or absence of periodontal pathogens distinguish between subjects with chronic and aggressive periodontitis? A systematic review. Journal of clinical periodontology. 2002;29 Suppl 3:10-21; discussion 37-8.

12. Wang D, Kawashima Y, Nagasawa T, Takeuchi Y, Kojima T, Umeda M, et al. Elevated serum IgG titer and avidity to Actinobacillus actinomycetemcomitans serotype $\mathrm{c}$ in Japanese periodontitis patients. Oral Microbiol Immunol. 2005 Jun;20(3):172-9.

13. Page RC, Beatty P, Waldrop TC. Molecular basis for the functional abnormality in neutrophils from patients with generalized prepubertal periodontitis. Journal of periodontal research. 1987 May;22(3):182-3.
14. Van Dyke TE, Horoszewicz HU, Cianciola LJ, Genco RJ. Neutrophil chemotaxis dysfunction in human periodontitis. Infection and immunity. 1980 Jan;27(1):124-32.

15. Van Dyke TE, Levine MJ, Tabak LA, Genco RJ. Juvenile periodontitis as a model for neutrophil function: reduced binding of the complement chemotactic fragment, C5a. Journal of dental research. 1983 Aug;62 (8):870-2.

16. Kinane DF, Cullen CF, Johnston FA, Evans CW. Neutrophil chemotactic behaviour in patients with early-onset forms of periodontitis (II). Assessment using the under agarose technique. Journal of clinical periodontology. 1989 Apr;16(4):247-51.

17. Takahashi K, Ohyama H, Kitanaka M, Sawa T, Mineshiba J, Nishimura F, et al. Heterogeneity of host immunological risk factors in patients with aggressive periodontitis. Journal of periodontology. 2001 Apr;72(4): 425-37.

18. Daniel MA, McDonald G, Offenbacher S, Van Dyke TE. Defective chemotaxis and calcium response in localized juvenile periodontitis neutrophils. Journal of periodontology. 1993 Jul;64(7):617-21.

19. Van Dyke TE, Schweinebraten M, Cianciola LJ, Offenbacher S, Genco RJ. Neutrophil chemotaxis in families with localized juvenile periodontitis. Journal of periodontal research. 1985 Sep;20(5):503-14.

20. Picolos DK, Lerche-Sehm J, Abron A, Fine JB, Papapanou PN. Infection patterns in chronic and aggressive periodontitis. Journal of clinical periodontology. 2005 Oct;32(10):1055-61.

21. Gunsolley JC, Pandey JP, Quinn SM, Tew J, Schenkein HA. The effect of race, smoking and immunoglobulin allotypes on IgG subclass concentrations. Journal of periodontal research. 1997 May;32(4):381-7.

22. Albandar JM, DeNardin AM, Adesanya MR, Winn DM, Diehl SR. Associations of serum concentrations of IgG, $\operatorname{IgA}, \operatorname{IgM}$ and interleukin-l beta with early-onset periodontitis classification and race. Journal of clinical periodontology. 2002 May;29(5):421-6.

23. AAP. Parameter on aggressive periodontitis. American Academy of Periodontology. J Periodontol. 2000 May;71(5 Suppl):867-9.

\section{CORRESPONDENCIA}

Nicola Discepoli

Correo electrónico: discepoli_nicola@hotmail.com 